\title{
Prognostic Value of Phase-Contrast Cine-Magnetic Resonance Imaging-Derived Global Coronary Flow Reserve in Patients With Non-ST-Segment Elevation Acute Coronary Syndrome Treated With Urgent Percutaneous Coronary Intervention
}

\author{
Yoshihisa Kanaji, MD; Taishi Yonetsu, MD; Rikuta Hamaya, MD; Tadashi Murai, MD; \\ Eisuke Usui, MD; Masahiro Hoshino, MD; Masao Yamaguchi, MD; Masahiro Hada, MD; \\ Yoshinori Kanno, MD; Hiroaki Ohya, MD; Youhei Sumino, MD; Hidenori Hirano, MD; \\ Haruhito Yuki, MD; Tomoki Horie, MD; Akinori Sugano, MD; Tetsumin Lee, MD; \\ Kenzo Hirao, MD, PhD; Tsunekazu Kakuta, MD, PhD
}

\begin{abstract}
Background: Phase-contrast cine-magnetic resonance imaging (PC-CMR) of the coronary sinus (CS) is a promising approach for quantifying coronary sinus flow (CSF) and global coronary flow reserve (G-CFR). We evaluated the prognostic value of G-CFR using PC-CMR in patients with non-ST-segment elevation acute coronary syndrome (NSTE-ACS).

Methods and Results: The study prospectively enrolled 116 NSTE-ACS patients who underwent uncomplicated urgent $\mathrm{PCl}$ within $48 \mathrm{~h}$ of symptom onset. Post-PCI (median, 20 days) PC-CMR images of the CS were acquired to assess absolute CSF at rest and during maximum hyperemia. The association of G-CFR with major adverse cardiac events (cardiac death, nonfatal myocardial infarction, late revascularization, or hospitalization for congestive heart failure) was investigated. Rest and maximal hyperemic CSF and corrected G-CFR were 1.27 [interquartile range, $0.79-1.73$ ] $\mathrm{mL} / \mathrm{min} / \mathrm{g}, 2.95$ [2.02-3.84] mL/min/g, and 2.42 [1.69-3.34], respectively. At a median follow-up of 17 months, cardiac event-free survival was significantly worse in patients with a corrected G-CFR $<2.33$ (log-rank $\left.X^{2}=19.5, \mathrm{P}<0.001\right)$. Cox proportional-hazards analysis showed that corrected G-CFR (hazard ratio, $0.434,95 \% \mathrm{Cl}, 0.270-$ $0.699, \mathrm{P}<0.001$ ) and NT-pro BNP at admission (hazard ratio, $1.0001,95 \% \mathrm{Cl}, 1.0000-1.0001, \mathrm{P}=0.007$ ) were independent predictors of adverse cardiac events during follow-up.
\end{abstract}

Conclusions: In NSTE-ACS patients successfully revascularized within $48 \mathrm{~h}$ of onset, post-PCI PC-CMR-derived G-CFR provided significant prognostic information independent of infarct size and conventional risk scores.

Key Words: Cardiac magnetic resonance; Coronary flow reserve; Non-ST-segment elevation myocardial infarction; Percutaneous coronary intervention

$\mathbf{N}$ on-ST-segment elevation myocardial infarction (NSTEMI) comprises about one-third of all cases of acute coronary syndrome (ACS), showing a wide heterogeneity in its clinical presentation. ${ }^{1}$ Although early mortality is generally lower in NSTEMI than in STsegment elevation myocardial infarction (STEMI), this difference disappears at 1 year, and long-term mortality is higher in NSTEMI. This is likely because of differences in baseline characteristics, including older age, and more comorbidities in the non-ST-segment elevation ACS (NSTE-ACS) population than in STEMI patients. ${ }^{24}$ Although early revascularization is reported to be associ- ated with improved long-term outcomes for NSTE-ACS and STEMI, NSTE-ACS is still associated with higher rates of major adverse cardiac events (MACE) compared with STEMI, even after successful revascularization., $2, \mathbf{6}$ Given this background, prognostic tools for identifying NSTE-ACS patients at high risk for worse long-term outcomes after percutaneous coronary intervention (PCI) are crucial.

Cardiac magnetic resonance imaging (CMR) provides multifaceted clinical information, including cardiac function, chamber size, regional wall motion abnormalities, infarct size, and microvascular obstruction. Recent studies

Received November 8, 2018; revised manuscript received February 21, 2019; accepted March 6, 2019; J-STAGE Advance Publication released online April 17, 2019 Time for primary review: 40 days

Cardiovascular Medicine, Tsuchiura Kyodo General Hospital, Tsuchiura (Y. Kanaji, R.H., T.M., E.U., M. Hoshino, M.Y., M. Hada, Y. Kanno, H.O., Y.S., H.H., H.Y., T.H., A.S., T.K.); Cardiovascular Medicine, Tokyo Medical and Dental University, Tokyo (T.Y., T.L., K.H.), Japan

Mailing address: Tsunekazu Kakuta, MD, PhD, Department of Cardiovascular Medicine, Tsuchiura Kyodo General Hospital, 4-1-1 Otsuno, Tsuchiura 300-0028, Japan. E-mail: kaz@joy.E-mail.ne.jp

ISSN-1346-9843 All rights are reserved to the Japanese Circulation Society. For permissions, please e-mail: cj@j-circ.or.jp 
have shown that coronary flow reserve (CFR) assessed by positron emission tomography (PET) is associated with future cardiovascular events. ${ }^{7}$ Phase-contrast cine-CMR (PC-CMR) allows noninvasive quantification of coronary sinus (CS) blood flow (CSF) without the need for ionizing radiation, radioactive tracers, gadolinium, or intravascular catheterization. ${ }^{\mathbf{8} 9}$ The CS drains approximately $96 \%$ of the total myocardial blood flow (MBF) and a good correlation has been shown between MBF and CSF. ${ }^{10} \mathrm{PC}-\mathrm{CMR}$ of the CS for quantifying absolute coronary blood flow and CS flow reserve (G-CFR) has also been validated against PET. ${ }^{8,11}$ Thus, this study aimed to investigate CSF and G-CFR after uncomplicated PCI for NSTE-ACS and the prognostic value of these noninvasive physiological indices for identifying patients with worse prognoses during follow-up. We hypothesized that impaired G-CFR after uncomplicated urgent PCI might predict the occurrence of MACE during follow-up.

\section{Methods}

The data, analytical methods, and study materials will be made available to other researchers for the purposes of reproducing the results or replicating the procedure. The data that support the findings of this study are available from the corresponding author on reasonable request.

\section{Study Population}

This study was conducted at Tsuchiura Kyodo General Hospital and prospectively enrolled patients with NSTEACS who underwent PCI between August 2014 and January 2018 . We included patients aged at least 20 years who were admitted to the intensive care unit with a diagnosis of NSTE-ACS within $48 \mathrm{~h}$ of the last appearance of symptoms suggestive of myocardial ischemia and/or ST-T segment change in at least 2 leads, and who underwent successful PCI with an early invasive strategy $<48 \mathrm{~h}$ after admission. ${ }^{5}$ We included patients with unstable angina pectoris and NSTEMI when the single culprit lesion was identifiable and considered suitable for PCI. We excluded patients with significant left main coronary artery disease (CAD), chronic total occlusion, unidentifiable culprit lesions, significant valvular disease, previous coronary artery bypass grafting (CABG), MI within the past month, significant arrhythmia, renal insufficiency with a baseline serum creatinine level $>1.5 \mathrm{mg} / \mathrm{dL}$, and contraindication to CMR (e.g., pacemaker, internal defibrillator or other incompatible intracorporeal foreign bodies, pregnancy, and claustrophobia). Patients with visible side branch occlusion $(>1.5 \mathrm{~mm})$ and those with ST-T segment elevation after PCI were also excluded. Patients with a Thrombolysis in Myocardial Infarction (TIMI) flow grade 2 after PCI were included if they met none of the exclusion criteria. We defined successful and uncomplicated PCI as a reduction of the minimum stenosis diameter less than $20 \%$ without the no-reflow phenomenon (TIMI flow grade risk score of 0 or 1) and clinical improvement of symptoms and/or ST-T segment change.

Patients with multivessel CAD, who exhibited additional angiographic stenosis $\geq 50 \%$ in at least 1 coronary artery other than the culprit vessel were eligible for inclusion. When the non-infarct-related coronary arteries had lesions with diameter stenosis of $30-90 \%$ by visual estimation, the fractional flow reserve (FFR) was measured. Non-culprit lesions were considered significant in the case of either FFR $\leq 0.80$ or a visually assessed diameter stenosis $\geq 90 \%$, and these significant non-infarct-related artery stenoses were considered as candidates for revascularization at the time of the index primary PCI or as a planned staged procedure during the index hospitalization. Unsuitable lesions for PCI, including heavily calcified lesions, diffuse lesions, and lesions with small subtended myocardial mass, were left untreated on consensus of the institutional heart team. The second-stage procedure was performed between 3 and 7 days after the index procedure. CMR imaging was performed after non-infarct-related lesion revascularization in all patients. The study protocol agreed with the Declaration of Helsinki and was approved by the institutional ethics committee. All patients provided written informed consent before enrollment in this study.

\section{Cardiac Catheterization}

Invasive coronary angiography (CAG) and revascularization of the infarct-related artery were performed with ad hoc PCI via the routine use of drug-eluting stents (DES) with a 6-Fr system. Before the PCI procedure, all patients received a loading dose of $200 \mathrm{mg}$ aspirin and $300 \mathrm{mg}$ clopidogrel or $20 \mathrm{mg}$ prasugrel. Quantitative CAG analyses were performed using dedicated offline software (QAngio XA 7.3; Medis, Leiden, The Netherlands). All patients received a bolus injection of heparin (10,000 IU) preprocedurally, an additional bolus injection of 2,000 IU every hour if the procedure required more than $1 \mathrm{~h}$, and underwent DES implantation with balloon predilatation. The stent type and procedure strategy selected were at the operator's discretion. To avoid aggressive stent expansion, online quantitative CAG was used to determine the correct stent size. After reperfusion therapy, standard dual antiplatelet therapy was started according to current guidelines. All patients were instructed to strictly refrain from ingesting caffeinated beverages after admission. FFR was determined using a Radi Analyzer Xpress instrument with a Certus coronary pressure wire (St. Jude Medical, St. Paul, MN, USA), as described previously. FFR was calculated as the ratio of the mean distal coronary pressure to the mean aortic pressure during stable hyperemia induced by intravenous adenosine $(140 \mu \mathrm{g} / \mathrm{kg} / \mathrm{min}$ through a central vein).

\section{Biochemical Tests}

Biochemical measurements were performed at admission. High-sensitivity cardiac troponin-I (hs-cTnI) was measured using the ARCHITECT $i 2000$ SR STAT hs-cTnI assay (Abbott Laboratories, North Chicago, IL, USA); the lower limit of detection was $1.5 \mathrm{ng} / \mathrm{L}$. N-terminal pro-Btype natriuretic peptide (NT-proBNP) levels were determined using the Elecsys proBNP assay (Roche Diagnostics, Basel, Switzerland); the lower limit of detection was $5 \mathrm{ng} / \mathrm{L}$.

\section{CMR Examination}

CMR Acquisition and Cine-CMR CMR image acquisition was performed using a 1.5-Tesla scanner (Philips Achieva, Philips Medical Systems, Best, The Netherlands) with 32-channel cardiac coils at 20 days [interquartile range, 16-28 days] after PCI. Cardiac gating and heart rate recording were achieved by vector-cardiogram device. Blood pressure (BP) was monitored throughout the protocol. Cine-CMR was performed using a retrospectively gated steady-state free precession sequence. The 12 short-axis slices of the left ventricle (LV) were acquired from the apex 


\begin{tabular}{|c|c|c|c|c|}
\hline & $\begin{array}{c}\text { Total } \\
(n=110)\end{array}$ & $\begin{array}{c}\text { MACE (+) } \\
(n=23)\end{array}$ & $\begin{array}{c}\text { MACE (-) } \\
(n=87)\end{array}$ & $P$ value \\
\hline \multicolumn{5}{|l|}{ Demographics } \\
\hline Age, years & $67 \pm 11$ & $66 \pm 12$ & $67 \pm 11$ & 0.61 \\
\hline Male, n (\%) & $90(81.8)$ & $16(69.6)$ & $74(85.1)$ & 0.13 \\
\hline $\mathrm{BMI}, \mathrm{kg} / \mathrm{m}^{2}$ & $24.3 \pm 3.2$ & $24.1 \pm 2.6$ & $24.4 \pm 3.4$ & 0.67 \\
\hline \multicolumn{5}{|l|}{ Medical history, n (\%) } \\
\hline MI & $10(9.1)$ & $2(8.7)$ & $8(9.2)$ & 1.00 \\
\hline $\mathrm{PCl}$ & $14(12.7)$ & $4(17.4)$ & $10(11.5)$ & 0.49 \\
\hline Hypertension & $74(67.2)$ & $16(69.6)$ & $58(66.7)$ & 1.00 \\
\hline Hyperlipidemia & $53(48.2)$ & $11(47.8)$ & $42(48.3)$ & 1.00 \\
\hline Diabetes mellitus & $37(33.6)$ & $11(47.8)$ & $26(29.9)$ & 0.14 \\
\hline Current smoker & $40(36.4)$ & $10(43.5)$ & $30(34.5)$ & 0.47 \\
\hline Family history & $11(10.0)$ & $3(13.0)$ & $8(9.2)$ & 0.70 \\
\hline \multicolumn{5}{|l|}{ NSTE-ACS presentation } \\
\hline Killip class & & & & 0.004 \\
\hline 1 & $98(89.0)$ & $16(69.6)$ & $82(94.3)$ & \\
\hline II & $6(5.5)$ & $4(17.4)$ & $2(2.3)$ & \\
\hline III & $5(4.5)$ & $2(8.7)$ & $3(3.4)$ & \\
\hline IV & $1(0.9)$ & $1(4.3)$ & $0(0)$ & \\
\hline Elevated troponin at presentation, $\mathrm{n}(\%)$ & $88(80.0)$ & $20(86.7)$ & $68(78.2)$ & 0.56 \\
\hline GRACE score & $128 \pm 36$ & $142 \pm 45$ & $124 \pm 32$ & 0.025 \\
\hline \multicolumn{5}{|l|}{ Coronary angiography } \\
\hline TIMI flow grade at baseline & & & & 0.66 \\
\hline 0 & $20(18.2)$ & $6(26.1)$ & $14(16.1)$ & \\
\hline 1 & $7(6.4)$ & $1(4.3)$ & $6(6.9)$ & \\
\hline 2 & $38(34.5)$ & $6(26.1)$ & $32(36.8)$ & \\
\hline 3 & $43(41.8)$ & $10(43.5)$ & $36(41.4)$ & \\
\hline TIMI flow grade at final & & & & 0.21 \\
\hline 0 & $0(0)$ & $0(0)$ & $0(0)$ & \\
\hline 1 & $0(0)$ & $0(0)$ & $0(0)$ & \\
\hline 2 & $10(9.1)$ & $4(17.4)$ & $6(6.9)$ & \\
\hline 3 & $100(90.9)$ & $19(82.6)$ & $81(93.1)$ & \\
\hline Culprit lesion location: RCA/LAD/LCx & $30 / 43 / 37$ & $9 / 9 / 5$ & $21 / 34 / 32$ & 0.27 \\
\hline Multivessel disease, n (\%) & $56(50.9)$ & $15(65.2)$ & $41(47.1)$ & 0.16 \\
\hline Staged $\mathrm{PCl}$ of the non-infarct-related artery during index hospitalization, $\mathrm{n}(\%)$ & $32(29.1)$ & $9(39.1)$ & $23(26.4)$ & 0.30 \\
\hline Ad hoc $\mathrm{PCl}$ of the non-infarct-related artery during index procedure, $\mathrm{n}(\%)$ & $11(10.0)$ & $2(8.7)$ & $9(10.3)$ & 1.00 \\
\hline \multicolumn{5}{|l|}{ Biochemical data } \\
\hline $\mathrm{TC}, \mathrm{mg} / \mathrm{dL}$ & $189[164,214]$ & $193[155,218]$ & $188[167,213]$ & 0.79 \\
\hline LDL-C, mg/dL & $115[94,142]$ & $118[90,151]$ & $114[96,140]$ & 0.73 \\
\hline $\mathrm{HDL}-\mathrm{C}, \mathrm{mg} / \mathrm{dL}$ & $45[39,51]$ & $47[40,50]$ & $45[38,51]$ & 0.54 \\
\hline $\mathrm{TG}, \mathrm{mg} / \mathrm{dL}$ & $124[83,175]$ & $120[78,160]$ & $125[86,180]$ & 0.52 \\
\hline Creatinine, $\mathrm{mg} / \mathrm{dL}$ & $0.79[0.69,0.97]$ & $0.81[0.72,0.99]$ & $0.78[0.68,0.97]$ & 0.64 \\
\hline eGFR, $\mathrm{mL} / \mathrm{min} / 1.73 \mathrm{~m}^{2}$ & $71.6 \pm 18.5$ & $69.8 \pm 25.0$ & $72.1 \pm 16.6$ & 0.60 \\
\hline $\mathrm{HbA} 1 \mathrm{c}, \%$ & $6.1[5.7,6.8]$ & $6.3[5.7,7.6]$ & $6.0[5.7,6.7]$ & 0.15 \\
\hline NT-proBNP, ng/L & $394,[148,1,050]$ & $1,050[297,2,580]$ & $276[135,885]$ & 0.014 \\
\hline cTnl at presentation, $\mathrm{ng} / \mathrm{L}$ & $276[40,2,428]$ & $469[166,4,539]$ & $179[38,2,167]$ & 0.23 \\
\hline Peak CK, IU/L & $313[144,1,021]$ & $401[145,1,629]$ & $288[149,1,017]$ & 0.84 \\
\hline Peak CK-MB, IU/L & $33[14,106]$ & $39[16,153]$ & $30[14,98]$ & 0.39 \\
\hline \multicolumn{5}{|l|}{ Prescriptions at discharge, $\mathrm{n}(\%)$} \\
\hline ACEI or ARB & $98(89.1)$ & $20(87.0)$ & $78(89.7)$ & 0.71 \\
\hline$\beta$-blocker & $83(75.5)$ & $19(82.6)$ & $64(73.6)$ & 0.43 \\
\hline Nitrates & $17(15.5)$ & $6(26.1)$ & $11(12.6)$ & 0.19 \\
\hline Statin & $102(92.7)$ & $22(95.7)$ & $80(92.0)$ & 1.00 \\
\hline Calcium-channel blocker & $39(35.5)$ & $7(30.4)$ & $32(36.8)$ & 0.63 \\
\hline
\end{tabular}

Values are $\mathrm{n}(\%)$, mean $\pm \mathrm{SD}$ or median [interquartile range]. ACEI, angiotensin-converting enzyme inhibitor; ARB, angiotensin-receptor blocker; BMI, body mass index; CK-MB, creatine kinase myocardial bound; CTnl, cardiac troponin-I; eGFR, estimated glomerular filtration rate; GRACE, Global Registry of Acute Coronary Events; HDL-C, high-density lipoprotein cholesterol; LAD, left anterior descending; LCx, left circumflex artery; LDL-C, low-density lipoprotein cholesterol; MACE, major adverse cardiac event; MI, myocardial infarction; NSTE-ACS, non-ST-segment elevation acute coronary syndrome; NT-proBNP, N-terminal pro-B-type natriuretic peptide; $\mathrm{PCl}$, percutaneous coronary intervention; RCA, right coronary artery; TC, total cholesterol; TIMI, Thrombolysis in Myocardial Infarction. 

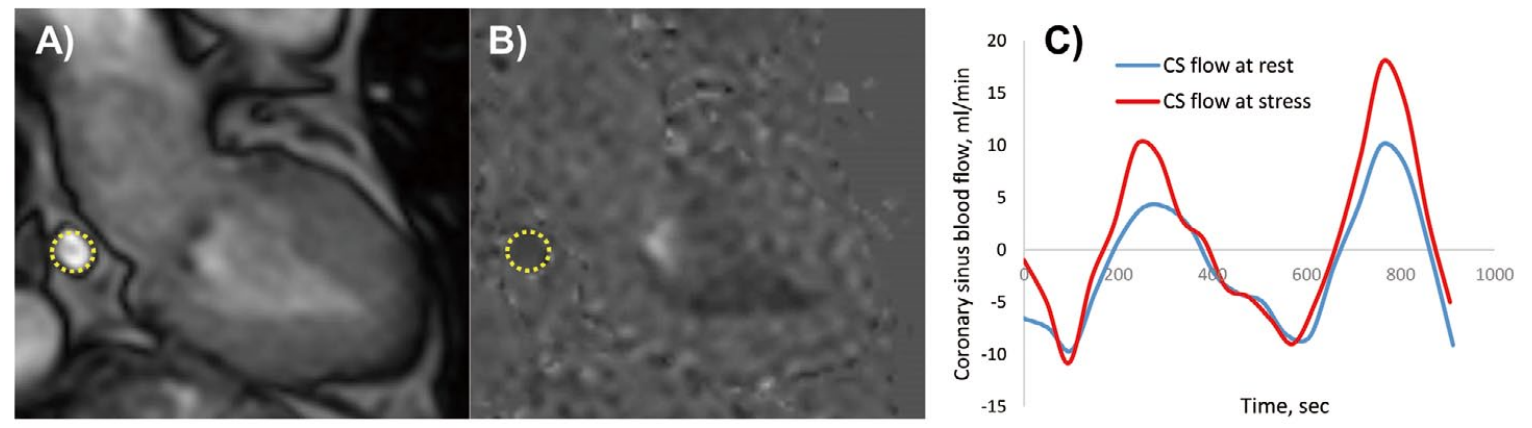

Figure 1. Phase-contrast cine-magnetic resonance images of the coronary sinus (CS). (A) Enlarged view of the CS with a dotted circle showing the outline. (B) Blood flow in the CS observed as a low-signal-intensity area. (C) Representative pattern of the CS blood flow curve. Blue line: resting flow; Red line: hyperemic flow.

to the base. The cine-CMR parameters were as follows: repetition time/echo time $4.1 \mathrm{~ms} / 1.4 \mathrm{~ms}$, slice thickness $6 \mathrm{~mm}$, flip angle $55^{\circ}$, field of view $350 \times 350 \mathrm{~mm}^{2}$, matrix size $128 \times 128$, and 20 phases per cardiac cycle. LV mass and volumes were calculated from Simpson's rule using CMR data. ${ }^{11}$

CSF Measurement The CS was identified in the atrioventricular groove using the basal slices of the short-axis stack. The plane for flow measurement by PC-CMR was positioned perpendicular to the CS at approximately $1-2 \mathrm{~cm}$ from the ostium. ${ }^{11}$ Velocity-encoded images were acquired with retrospective ECG-gating during approximately 15 -s breath holds and imaging parameters were: repetition time/echo time $7.3 \mathrm{~ms} / 4.4 \mathrm{~ms}$, flip angle $10^{\circ}$, field of view $250 \times 250 \mathrm{~mm}^{2}$, acquisition matrix $128 \times 128,20$ phases per cardiac cycle, encoding $50 \mathrm{~cm} / \mathrm{s}$, and slice thickness $6 \mathrm{~mm}$. PC-CMR of the CS measurements was accomplished during maximal hyperemia and at rest. Maximal stable hyperemia was induced by intravenous adenosine $(140 \mu \mathrm{g} / \mathrm{kg} / \mathrm{min}$ through a central venous catheter). The duration between the end of hyperemia and resting image acquisition was $10 \mathrm{~min}$. All patients were strictly instructed to refrain from caffeinated beverages for more than $24 \mathrm{~h}$ before CMR examination.

Late Gadolinium-Enhanced (LGE) Images After the acquisition of PC-CMR images, gadolinium contrast was injected intravenously at a total dose of $0.10 \mathrm{mmol} / \mathrm{kg}$; $15 \mathrm{~min}$ later the LGE images were acquired in the same planes as the cine images and imaging parameters were: repetition time/echo time $3.8 \mathrm{~ms} / 1.28 \mathrm{~ms}$, flip angle $15^{\circ}$, field of view $350 \times 350 \mathrm{~mm}^{2}$, acquisition matrix $200 \times 175,20$ phases per cardiac cycle, encoding $50 \mathrm{~cm} / \mathrm{s}$, and slice thickness $8 \mathrm{~mm}$.

\section{PC-CMR Image Analysis}

CSF quantitative analysis by PC-CMR was performed using proprietary software (Philips View Forum, Best, The Netherlands) in a blinded fashion by 2 expert researchers (Y.K, R.H). The CS contour was traced on the magnitude images throughout the cardiac cycle. CSF was quantified by integrating the flow rate values from each cardiac cycle and multiplying by the mean heart rate during the acquisition. CSF quantification was performed during maximum hyperemia and at rest. CSF at rest was corrected using rate-pressure products (RPP), as follows. ${ }^{12} \mathrm{RPP}=$ systolic $\mathrm{BP}(\mathrm{mmHg}) \times$ heart rate; corrected $\mathrm{CSF}=(\mathrm{CSF} / \mathrm{RPP}) \times 10,000$; and corrected $\mathrm{CSF}(\mathrm{mL} / \mathrm{min} / \mathrm{g})=$ corrected $\mathrm{CSF} / \mathrm{LV}$ mass (g). G-CFR was calculated by CSF during maximal hyperemia/CSF at rest. Ventricular volumes, ejection fraction $(\mathrm{EF})$, and LV mass were derived by contouring the endoand epicardial borders on the short-axis cine images. The amount of LGE was assessed using a threshold of 5 standard deviations above the signal intensity of normal myocardium as proposed by current recommendations. ${ }^{13}$ Microvascular obstruction was assessed as the hypointense areas on LGE images, as previously described. ${ }^{14}$

\section{Assessment of Outcomes}

Patients were followed up for the occurrence of MACE including cardiac death, MI, clinically driven remote $(>3$ months after the index PCI) revascularization, or hospitalization for congestive heart failure (HF). Cardiac death was defined as any death preceded by acute mitral regurgitation, decompensated HF, ventricular arrhythmia, or new acute MI or death preceded by mechanical complications of MI. Clinical endpoints were determined by the blinded assessment of hospital records or via telephone interviews.

\section{Statistical Analysis}

Statistical analysis was performed using SPSS version 23.0 (SPSS, Inc., Chicago, IL, USA). Categorical data are expressed as absolute frequencies and percentages and were compared using $\chi^{2}$ or Fisher exact tests, as appropriate. Continuous variables are expressed as mean \pm standard deviation for normally distributed variables or as median (25th-75th percentile) for non-normally distributed variables and were compared using Student's t-tests and the Mann-Whitney U-test, respectively. Receiver-operating curves were analyzed to assess the best cutoff values for predicting MACE. The optimal cutoff was calculated using Youden's index. Survival curves were estimated using Kaplan-Meier estimates and were compared using logrank tests. A Cox proportional-hazards regression model was used to identify independent predictors of MACE. The covariates with $\mathrm{P}<0.10$ in the univariate analysis were included in the multivariate analysis. As a risk index score, Killip class, not the Global Registry of Acute Coronary Events (GRACE) score, was entered in the multivariate model by considering the hazard ratio (HR) and collinearity. Intra- and interobserver variability of CSF assessment was assessed using intraclass correlation coefficients. 


\begin{tabular}{|c|c|c|c|c|}
\hline & $\begin{array}{c}\text { Total } \\
(n=110)\end{array}$ & $\begin{array}{c}\text { MACE (+) } \\
(n=23)\end{array}$ & $\begin{array}{c}\text { MACE (-) } \\
(n=87)\end{array}$ & $P$ value \\
\hline $\mathrm{EDV}, \mathrm{mL}$ & $124.8[106.5,147.0]$ & $133.2[112.1,157.6]$ & $123.2[106.2,145.9]$ & 0.20 \\
\hline $\mathrm{ESV}, \mathrm{mL}$ & $53.2[42.0,69.0]$ & $58.7[46.9,80.9]$ & $51.4[41.7,67.2]$ & 0.13 \\
\hline LVEF, \% & $57.4[50.1,63.4]$ & $54.0[42.0,58.4]$ & $58.2[50.4,64.2]$ & 0.035 \\
\hline LV mass, g & $102.5[89.2,125.2]$ & $104.8[87.1,126.1]$ & $101.7[89.3,119.2]$ & 0.78 \\
\hline LGE volume, $\mathrm{cm}^{3}$ & $4.0[0.7,7.4]$ & $4.4[1.5,12.8]$ & $3.9[0.63,7.2]$ & 0.25 \\
\hline MVO presence, $\mathrm{n}(\%)$ & $17(15.5)$ & $6(26.1)$ & $11(12.6)$ & 0.19 \\
\hline $\mathrm{CSF}$ at rest, $\mathrm{mL} / \mathrm{min}$ & $113.2[77.7,140.5]$ & $127.6[111.0,181.3]$ & $105.9[70.7,136.8]$ & 0.004 \\
\hline Corrected CSF at rest, $\mathrm{mL} / \mathrm{min}$ & $131.5[90.9,173.3]$ & $152.1[125.0,205.7]$ & $128.9[82.3,158.6]$ & 0.003 \\
\hline Rate-pressure products at rest, $\mathrm{mmHg} / \mathrm{min}$ & $8,702[7,670,9,800]$ & $8,636[7,969,9,919]$ & $8,710[7,670,9,734]$ & 0.75 \\
\hline CSF at hyperemia, $\mathrm{mL} / \mathrm{min}$ & $308.2[205.4,401.6]$ & $278.8[174.3,364.3]$ & $314.4[215.0,407.1]$ & 0.20 \\
\hline $\mathrm{CSF}$ at rest, $\mathrm{mL} / \mathrm{min} / \mathrm{g}$ & $1.10[0.74,1.47]$ & $1.38[1.06,1.58]$ & $0.99[0.67,1.38]$ & 0.003 \\
\hline Corrected CSF at rest, $\mathrm{mL} / \mathrm{min} / \mathrm{g}$ & $1.27[0.79,1.73]$ & $1.65[1.20,1.94]$ & $1.18[0.75,1.64]$ & 0.002 \\
\hline CSF at hyperemia, $\mathrm{mL} / \mathrm{min} / \mathrm{g}$ & $2.95[2.02,3.84]$ & $2.52[1.65,3.73]$ & $3.11[2.05,4.02]$ & 0.24 \\
\hline G-CFR & $2.86[1.91,3.89]$ & $1.83[1.19,2.30]$ & $3.12[2.25,4.07]$ & $<0.001$ \\
\hline Corrected G-CFR & $2.42[1.69,3.34]$ & $1.65[1.07,2.20]$ & $2.63[2.07,3.63]$ & $<0.001$ \\
\hline
\end{tabular}

Values are $\mathrm{n}(\%)$ or median [interquartile range]. CSF, coronary sinus flow; EDV, end-diastolic volume; EF, ejection fraction; ESV, end systolic volume; G-CFR, global coronary flow reserve; LGE, late gadolinium enhancement; MVO, microvascular obstruction. Other abbreviations as in Table 1.

\begin{tabular}{|c|c|}
\hline & $\begin{array}{c}\text { Total } \\
(n=23)\end{array}$ \\
\hline Cardiac death, $\mathrm{n}(\%)$ & $2(1.8)$ \\
\hline Nonfatal MI, n (\%) & $6(5.5)$ \\
\hline Hospitalization for HF, n (\%) & $4(3.6)$ \\
\hline \multicolumn{2}{|c|}{ Clinically driven revascularization, $\mathrm{n}(\%)$} \\
\hline $\mathrm{PCl}$ & $9(8.2)$ \\
\hline CABG & $2(1.8)$ \\
\hline
\end{tabular}

CABG, coronary artery bypass grafting; $\mathrm{HF}$, heart failure. Other abbreviations as in Table 1.

Reproducibility was also evaluated via Bland-Altman analysis. A two-sided $\mathrm{P}<0.05$ was considered statistically significant.

\section{Results}

\section{Patients' Characteristics}

Of 116 initially enrolled patients, 3 were excluded from the final analysis because of unsatisfactory PC-CMR data acquisition. One other patient could not complete the CMR examination because of atrioventricular block and bradycardia resulting from adenosine infusion, and another patient exhibited the no-reflow phenomenon after stent implantation with subsequent STEMI. These 5 patients were excluded from the study and so the PC-CMR analysis was performed for 111 NSTE-ACS patients who successfully underwent emergency PCI. Of these 111 patients, 1 was lost to follow-up and the final follow-up data of 110 patients ( $94.8 \%$ of initially enrolled patients) were analyzed. There were no significant complications related to the CMR examination in these patients. Their baseline characteristics and angiographic findings are summarized in Table $\mathbf{1}$ according to the presence or absence of MACE. In patients with MACE, Killip class, GRACE score, and NT-proBNP levels at presentation were signifi- cantly higher compared with those without MACE (all $\mathrm{P}<0.05)$ (Table 1). At presentation, 88 patients $(80.0 \%)$ showed elevated hs-cTnI (institutional upper reference limit: $70 \mathrm{ng} / \mathrm{L}$ ) and 9 patients exhibited cTnI elevation above the upper reference limit immediately before emergency catheterization. Thus, 97 patients $(88.2 \%)$ met the institutional criteria of NSTEMI, while 13 were classified as unstable angina pectoris at the time of PCI. In 11 patients, non-infarct-related artery stenoses were subsequently treated with PCI at the time of index primary PCI according to the results of FFR examination or angiographically severe diameter stenosis $>90 \%$. Staged PCI for non-infarct-related artery lesions was performed in 32 patients out of the 45 remaining with multivessel disease. In 13 patients with multivessel disease, revascularization was not performed by consensus of the institutional heart team. No significant difference in the prevalence of untreated lesions between the 2 groups with and without MACE was observed. All patients in the present study were considered to be properly revascularized before CMR examination in the daily clinical setting. In the present cohort, no patient underwent $\mathrm{CABG}$ on the basis of the index $\mathrm{CAG}$ and PCI results.

\section{CMR Findings}

Figure 1 shows a representative case of CSF measurements by PC-CMR. CMR results are shown in Table 2. In the total cohort, CSF was 1.27 [0.79-1.73] and 2.95 [2.02-3.84] $\mathrm{mL} / \mathrm{min} / \mathrm{g}$ at rest and during maximum hyperemia, respectively. Microvascular obstruction was detected in $9(8.2 \%)$ patients and the median LGE volume was 4.0 [0.7-7.4] $\mathrm{cm}^{3}$. LVEF and G-CFR were significantly lower, and CSF at rest was higher in patients with MACE $(\mathrm{P}=0.035$, $\mathrm{P}<0.001$, and $\mathrm{P}<0.01$, respectively) compared with those without MACE, but no difference between groups was observed in CSF at hyperemia.

\section{Outcome Data}

During follow-up (514 [281-1,137] days), cardiac death occurred in 2 patients $(1.8 \%)$ and 4 patients $(3.6 \%)$ were 


\begin{tabular}{|c|c|c|c|c|c|c|}
\hline & \multicolumn{3}{|c|}{ Univariable analysis } & \multicolumn{3}{|c|}{ Multivariable analysis } \\
\hline & $P$ value & HR & $95 \% \mathrm{Cl}$ & $P$ value & HR & $95 \% \mathrm{Cl}$ \\
\hline NT-proBNP (ng/L) & 0.001 & 1.0001 & $1.0000-1.0001$ & 0.007 & 1.0001 & $1.0000-1.0001$ \\
\hline Max. CK-MB (IU/L) & 0.11 & 1.003 & $1.000-1.005$ & & & \\
\hline cTnl at presentation & 0.57 & 0.99 & $0.94-1.03$ & & & \\
\hline Killip class at presentation & 0.002 & 4.10 & $1.69-10.0$ & 0.34 & 1.65 & $0.597-4.549$ \\
\hline GRACE score & 0.037 & 1.01 & $1.00-1.03$ & & & \\
\hline Multivessel disease & 0.12 & 1.99 & $0.85-4.68$ & & & \\
\hline $\mathrm{PCl}$ of the non-culprit artery & 0.18 & 1.81 & $0.77-4.27$ & & & \\
\hline LVEF & 0.14 & 0.98 & $0.95-1.01$ & & & \\
\hline LGE volume $\left(\mathrm{cm}^{3}\right)$ & 0.13 & 1.04 & $0.99-1.09$ & & & \\
\hline CSF reserve (corrected) & $<0.001$ & 0.41 & $0.25-0.65$ & $<0.001$ & 0.434 & $0.270-0.699$ \\
\hline
\end{tabular}

$\mathrm{Cl}$, confidence interval; LVEF, left ventricular ejection fraction. Other abbreviations as in Tables 1,2.

\section{A) Corrected G-CFR}

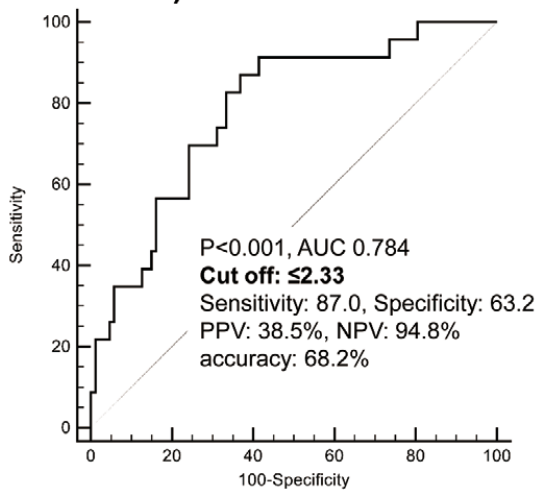

B) NT-pro BNP

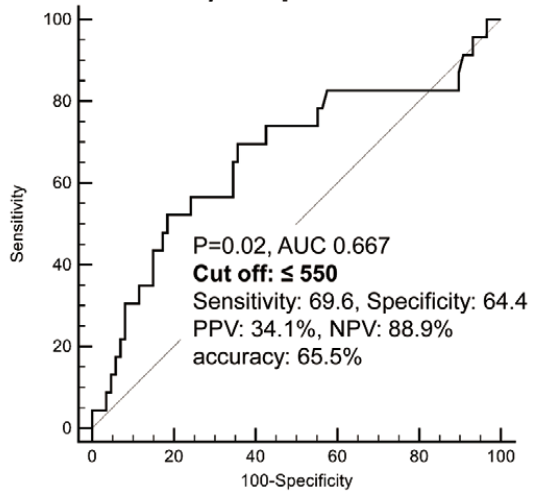

C) Frequency of MACE

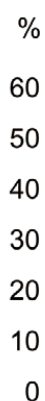

$\%$

60

50

40

30

20

10

0

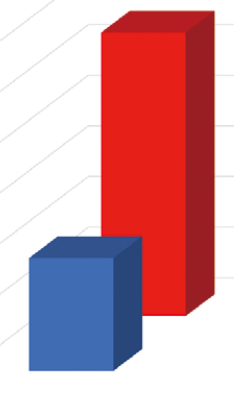

$<2.33$

G-CFR
Figure 2. Receiver-operating characteristic curve analyses for determining the optimal cutoff value of $(\mathbf{A})$ corrected G-CFR and (B) NT-proBNP for predicting MACE. A lower G-CFR consistently identified higher-risk patients in both categories of NT-pro BNP level. (C) Frequency of MACE by categories of corrected G-CFR and NT-pro BNP level. AUC, area under the curve; G-CFR, global coronary flow reserve; MACE, major adverse cardiac events; NPV, negative predictive value; NT-proBNP, $\mathrm{N}$-terminal pro-B-type natriuretic peptide; PPV, positive predictive value. hospitalized for HF. Clinically driven remote revascularization was performed in 11 patients $(10.0 \%)$ : $2(1.8 \%)$ treated by CABG and $9(8.2 \%)$ treated with PCI, respectively. Among them, target lesion-related PCI was performed in $4(3.6 \%)$ patients (Table 3).

\section{Survival Analysis}

There was no difference in median follow-up between patients with and without MACE. There was no difference in the length of the index hospital stay between those with and without $\operatorname{MACE}(9[7,16]$ vs. $8[7,12]$ days, $\mathrm{P}=0.25)$. The baseline characteristics and angiographic findings, including procedural variables and CMR findings are summarized in Table 1 and Table 2. All patients had high rates of optimal medical therapy during hospital stay, at discharge, and during follow-up, with no differences observed between groups. Stepwise multivariable Cox regression proportional-hazards analysis showed that the post-PCI G-CFR and NT-proBNP level at admission remained independent predictors of adverse cardiac events during 


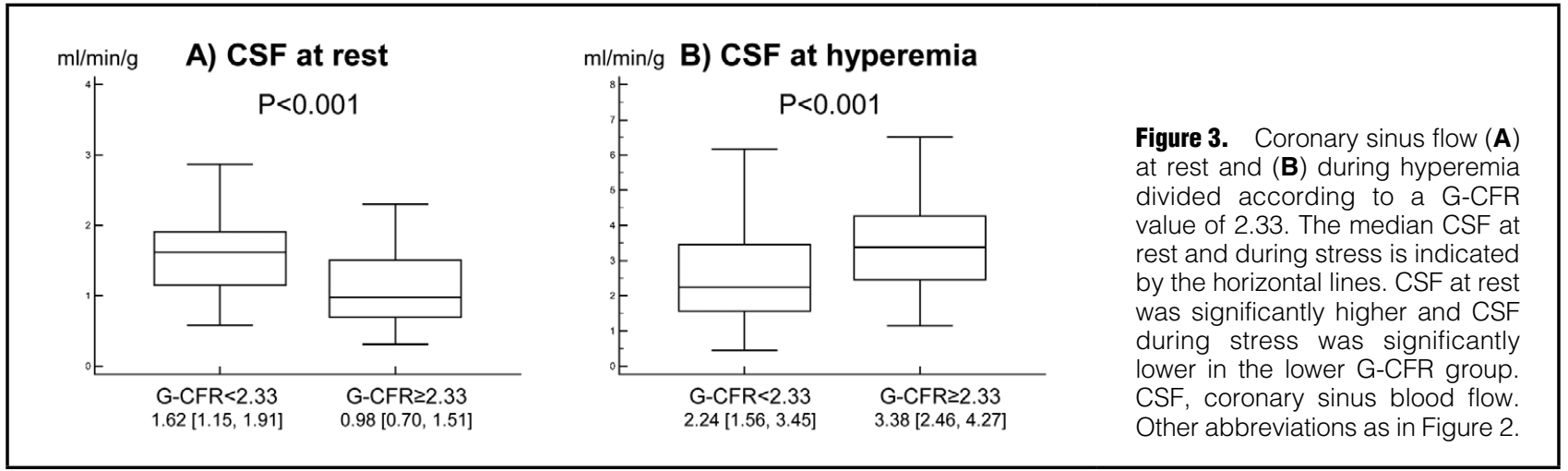

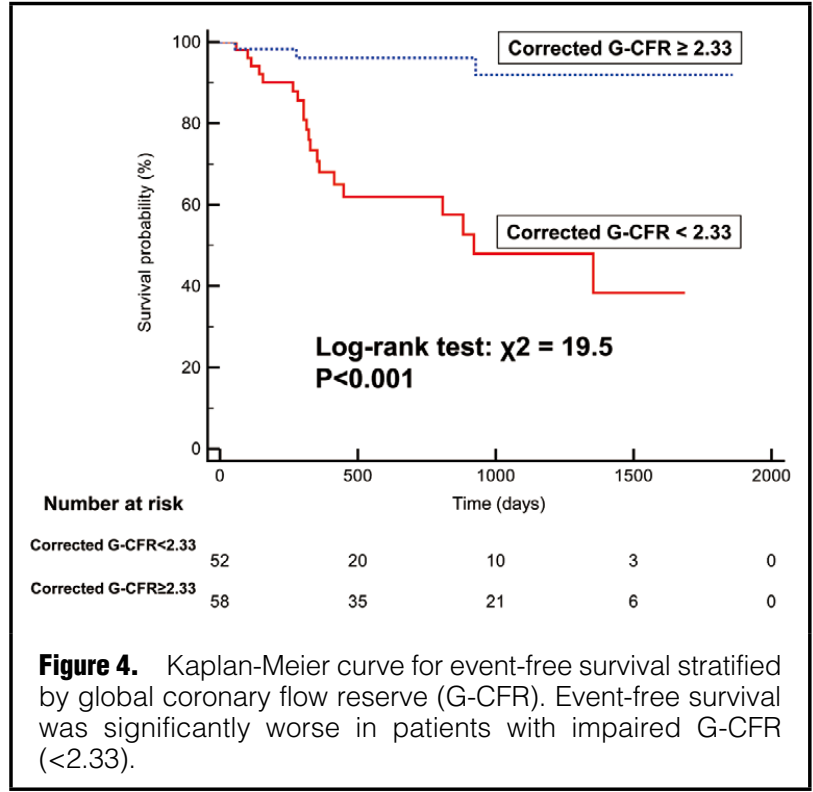

follow-up (Table 4).

Receiver-operating characteristic curve analysis revealed that the optimal cutoff values of corrected G-CFR and NT-pro BNP level for predicting adverse events were 2.33 (area under the curve [AUC] $0.784 ; 95 \%$ confidence interval [CI] 0.695-0.856) for G-CFR and $550 \mathrm{ng} / \mathrm{L}$ (AUC $0.667 ; 95 \%$ CI $0.570-0.754$ ) for NT-proBNP level (Figure 2A,B). Lower G-CFR consistently identified higher-risk patients at each category of NT-pro BNP level (Figure 2C). When divided into 2 groups by the G-CFR value of 2.33, CSF at rest was significantly higher and CSF at stress was significantly lower in the group with a lower G-CFR $(\mathrm{P}<0.001$ and $\mathrm{P}<0.001$, respectively) (Figure 3).

Survival from MACE was significantly worse in patients with G-CFR $<2.33$ (log-rank $\chi^{2}=19.5, \mathrm{P}<0.001$, Figure 4). G-CFR $<2.33$ also significantly predicted MACE in the subgroup analysis limited to the 1-vessel disease subgroup $(\mathrm{n}=54)\left(\log\right.$-rank $\left.\chi^{2}=9.2, \mathrm{P}=0.003\right)$.

\section{CSF Reproducibility}

The reproducibility of CSF measurements was satisfactory in the interobserver (intraclass correlation: 0.82 for CSF at rest) and intraobserver (intraclass correlation: 0.85 ) reproducibility analysis. Supplementary Figure shows the Bland-
Altman analysis of interobserver reproducibility of CSF during maximal hyperemia for first 50 cases.

\section{Discussion}

The important findings of the present study were as follows. In patients with NSTE-ACS who underwent successful emergency PCI by early invasive strategy and subsequent residual lesion revascularization, (1) CSF, which estimates absolute LV MBF, was successfully measured by PC-CMR without significant complications in a series of 111 patients $(95.7 \%)$ at rest and during maximal hyperemia after emergency PCI; (2) G-CFR was noninvasively obtained without the need for contrast medium, radioactive tracers, ionizing radiation, or intravascular catheterization, based on the CSF measured by PC-CMR early after DES implantation; and (3) impaired G-CFR and increased NT-pro BNP were independently associated with worse outcomes. To our knowledge, this is the first study to report the prognostic efficacy of G-CFR as a surrogate of global MBF reserve obtained by PC-CMR in NSTE-ACS patients early after emergency PCI.

Recent studies have examined the prognostic efficacy of noninvasive estimates of CFR to predict adverse outcomes. ${ }^{7,15} \mathrm{CFR}$ is a well-validated index that allows assessment of impaired coronary blood flow caused by obstructive, diffuse, or microcirculatory involvement of the coronary circulation and has been demonstrated to improve risk stratification not only in patients with demonstrable ischemia and/or scar, but also among patients with normal perfusion. PC-CMR of CS allows noninvasive quantification of LV MBF and G-CFR, which have been validated against PET.11,16 PET-derived $\mathrm{MBF}$ and CFR are known predictors for future adverse events in patients with stable CAD. ${ }^{17,18}$ On the other hand, G-CFR derived from CMR had no significant relationship with regional CFR derived by pressure wire in our previous study, ${ }^{19}$ which may indicate heterogeneity of coronary flow and microcirculation in patients with CAD. The prognostic implications of MBF and CFR have been validated mainly by PET studies in patients with stable CAD, ${ }^{17,18}$ and the clinical utility in ACS patients is of interest, and remains to be determined, because radiation is not required, cost is not high, and it takes less than $15 \mathrm{~min}$ to perform, giving better resource availability. Our results are in line with those of these studies and further demonstrated that G-CFR derived from PC-CMR of CSF after early revascularization therapy in patients with NSTE-ACS predicted 
event-free survival. Although the degree of myocardial necrosis was much less in the present study than is commonly observed in STEMI patients and all patients in this study were considered to be properly revascularized before CMR imaging, the post-PCI G-CFR values were widely distributed and impaired G-CFR was not uncommon. Our results suggested that, in addition to myocardial damage evaluated by an increase in the levels of cardiac markers or LGE volumes, other factors might affect global microvascular dysfunction, represented as low G-CFR after uncomplicated emergency PCI for NSTE-ACS and in the absence of significant epicardial stenosis suitable for PCI. In recent years, it has been acknowledged that not only an open epicardial artery but also myocardial perfusion is critical to myocardial salvage in the treatment of ACS and that well-preserved myocardial microcirculation is related to better prognosis. ${ }^{20}$ Our finding that G-CFR had prognostic efficacy may be related to cardiovascular dysfunction or excessive atherosclerotic burden, including diffuse disease and microvascular dysfunction that has extended beyond the damaged myocardium of the index infarction. Microvascular function reflects metabolic need, structural changes, autonomic innervation, and endothelial function. In fact, in the past 2 decades, studies have reported that abnormalities in the coronary microcirculation occur in many clinical conditions, including diabetes mellitus. ${ }^{21}$ Further, impaired vasodilatory function or microvascular dysfunction can cause myocardial ischemia and impaired G-CFR may influence myocardial contractility. LV dysfunction can also be influenced by global myocardial perfusion abnormalities, as previously reported. ${ }^{22}$

Our study extended the prognostic significance of G-CFR and showed that G-CFR, as estimated by PCCMR, was associated with worse outcomes in NSTE-ACS patients after revascularization. Our results additionally indicated that NT-proBNP provided independent and significant prognostic information in NSTE-ACS patients treated by early invasive management. Biomarkers are important in the diagnosis and management of ACS patients. Our results agree with those of previous studies, which indicated that NT-proBNP has prognostic significance. ${ }^{23}$ The present study demonstrated that NT-proBNP and G-CFR independently and incrementally improved risk discrimination early after NSTE-ACS beyond the TIMI risk score, GRACE score, troponin levels, and LVEF. Increased NT-proBNP is associated with atrial and ventricular distension via hemodynamic stress; therefore, it is conceivable that NT-proBNP is linked with an increased risk of $\mathrm{HF}$ and death. However, the mechanisms underlying the increased risk of new ischemic events associated with higher levels of NT-proBNP or lower G-CFR are elusive. The progression of coronary plaque and rupture or thrombus formation in coronary arteries might be more frequently accompanied with a greater systemic atherosclerotic burden and impaired endothelial function reflected by a lower CFR and LV functional impairment or increased end-diastolic pressures represented by increased NT-proBNP.

Although our results are hypothesis-generating, the stratification of NSTE-ACS patients based on post-PCI G-CFR obtained by PC-CMR of CS flow may provide important prognostic information after uncomplicated revascularization therapy. Moreover, the exclusion of ionizing radiation exposure and greater CMR availability supports its clinical application. Further large prospective population studies are required to confirm the efficacy of G-CFR in addition to NT-proBNP as a prognostic tool in NSTE-ACS patients at differing levels of clinical risk and potential for benefit of treatment.

\section{Study Limitations}

The results of the present study should be interpreted considering several important limitations. First, this study included a relatively small number of subjects from a single center. Rigorous exclusion criteria limited the number of the patients included in the study and may have resulted in selection bias. Further, patients were enrolled and excluded with the awareness of CMR contraindication and importance of ECG-gating, leading to a further selection bias as we had no patients with metallic device implants, bronchospasm, atrial fibrillation, or atrioventricular block. Although the measurement reliability was acceptable for assessing change in CSF, with the current spatial resolution of CMR, partial volume errors might have occurred, resulting in measurement errors. Baseline biomarker samplings were obtained at admission, resulting in individual differences in time from symptom onset to measurements. Although CMR imaging was performed after hemodynamic stabilization, central venous pressure and/or LV end-diastolic pressure might have influenced CSF measurements. We performed post-PCI PC-CMR examination at 20 days [16-28 days] after PCI. Different time windows of CMR and long-term changes in CSF after PCI should be evaluated in future studies.

\section{Conclusions}

G-CFR quantification using PC-CMR of CSF in NSTEACS patients after uncomplicated emergency PCI and residual lesion revascularization was feasible and may provide prognostic information. This technique does not require ionizing radiation, radioactive tracers, gadolinium, or intravascular catheterization. Because accurate risk stratification is essential in the heterogeneous population of NSTE-ACS for clinical decision-making and management, the results of this study provide additional potential insight for risk stratification of NSTE-ACS patients by demonstrating the prognostic significance of G-CFR obtained by PC-CMR of CSF.

\section{Sources of Funding / Disclosures}

None.

\section{References}

1. Avezum A, Makdisse M, Spencer F, Gore JM, Fox KA, Montalescot G, et al. Impact of age on management and outcome of acute coronary syndrome: Observations from the Global Registry of Acute Coronary Events (GRACE). Am Heart J 2005; 149: $67-73$.

2. Chan MY, Sun JL, Newby LK, Shaw LK, Lin M, Peterson ED, et al. Long-term mortality of patients undergoing cardiac catheterization for ST-elevation and non-ST-elevation myocardial infarction. Circulation 2009; 119: 3110-3117.

3. Goldberg RJ, Currie K, White K, Brieger D, Steg PG, Goodman $\mathrm{SG}$, et al. Six-month outcomes in a multinational registry of patients hospitalized with an acute coronary syndrome (the Global Registry of Acute Coronary Events [GRACE]). Am J Cardiol 2004; 93: 288-293.

4. Park HW, Yoon CH, Kang SH, Choi DJ, Kim HS, Cho MC, et al. Early- and late-term clinical outcome and their predictors in patients with ST-segment elevation myocardial infarction and non-ST-segment elevation myocardial infarction. Int J Cardiol 2013; 169: 254-261. 
5. Puymirat E, Taldir G, Aissaoui N, Lemesle G, Lorgis L, Cuisset $\mathrm{T}$, et al. Use of invasive strategy in non-ST-segment elevation myocardial infarction is a major determinant of improved longterm survival: FAST-MI (French Registry of Acute Coronary Syndrome). JACC Cardiovasc Interv 2012; 5: 893-902.

6. Cox DA, Stone GW, Grines CL, Stuckey T, Zimetbaum PJ, Tcheng JE, et al. Comparative early and late outcomes after primary percutaneous coronary intervention in ST-segment elevation and non-ST-segment elevation acute myocardial infarction (from the CADILLAC trial). Am J Cardiol 2006; 98: $331-337$

7. Taqueti VR, Hachamovitch R, Murthy VL, Naya M, Foster CR, Hainer $\mathbf{J}$, et al. Global coronary flow reserve is associated with adverse cardiovascular events independently of luminal angiographic severity and modifies the effect of early revascularization. Circulation 2015; 131: 19-27.

8. Koskenvuo JW, Hartiala JJ, Knuuti J, Sakuma H, Toikka JO, Komu M, et al. Assessing coronary sinus blood flow in patients with coronary artery disease: A comparison of phase-contrast MR imaging with positron emission tomography. Am J Roentgenol 2001; 177: 1161-1166.

9. Kato S, Saito N, Nakachi T, Fukui K, Iwasawa T, Taguri M, et al. Stress perfusion coronary flow reserve versus cardiac magnetic resonance for known or suspected CAD. J Am Coll Cardiol 2017; 70: 869-879.

10. Lund GK, Wendland MF, Shimakawa A, Arheden H, Stahlberg $\mathrm{F}$, Higgins $\mathrm{CB}$, et al. Coronary sinus flow measurement by means of velocity-encoded cine MR imaging: Validation by using flow probes in dogs. Radiology 2000; 217: 487-493.

11. Schwitter J, DeMarco T, Kneifel S, von Schulthess GK, Jorg $\mathrm{MC}$, Arheden H, et al. Magnetic resonance-based assessment of global coronary flow and flow reserve and its relation to left ventricular functional parameters: A comparison with positron emission tomography. Circulation 2000; 101: 2696-2702.

12. Murthy VL, Naya M, Taqueti VR, Foster CR, Gaber M, Hainer $\mathrm{J}$, et al. Effects of sex on coronary microvascular dysfunction and cardiac outcomes. Circulation 2014; 129: 2518-2527.

13. Schulz-Menger J, Bluemke DA, Bremerich J, Flamm SD, Fogel MA, Friedrich MG, et al. Standardized image interpretation and post processing in cardiovascular magnetic resonance: Society for Cardiovascular Magnetic Resonance (SCMR) board of trustees task force on standardized post processing. J Cardiovasc Magn Reson 2013; 15: 35.

14. Robbers LF, Eerenberg ES, Teunissen PF, Jansen MF, Hollander MR, Horrevoets AJ, et al. Magnetic resonance imaging-defined areas of microvascular obstruction after acute myocardial infarction represent microvascular destruction and haemorrhage. Eur Heart J 2013; 34: 2346-2353.

15. Herzog BA, Husmann L, Valenta I, Gaemperli O, Siegrist PT, Tay FM, et al. Long-term prognostic value of $13 \mathrm{~N}$-ammonia myocardial perfusion positron emission tomography added value of coronary flow reserve. J Am Coll Cardiol 2009; 54: 150-156.

16. van Rossum AC, Visser FC, Hofman MB, Galjee MA, Westerhof N, Valk J. Global left ventricular perfusion: Noninvasive measurement with cine MR imaging and phase velocity mapping of coronary venous outflow. Radiology 1992; 182: 685-691.

17. Taqueti VR, Shaw LJ, Cook NR, Murthy VL, Shah NR, Foster $\mathrm{CR}$, et al. Excess cardiovascular risk in women relative to men referred for coronary angiography is associated with severely impaired coronary flow reserve, not obstructive disease. Circulation 2017; 135: 566-577.

18. Murthy VL, Lee BC, Sitek A, Naya M, Moody J, Polavarapu V, et al. Comparison and prognostic validation of multiple methods of quantification of myocardial blood flow with $82 \mathrm{Rb}$ PET. $J$ Nucl Med 2014; 55: 1952-1958.

19. Kanaji Y, Yonetsu T, Hamaya R, Murai T, Usui E, Hoshino M, et al. Impact of elective percutaneous coronary intervention on global absolute coronary flow and flow reserve evaluated by phase-contrast cine-magnetic resonance imaging in relation to regional invasive physiological indices. Circ Cardiovasc Interv 2018; 11: e006676.

20. Gibson CM, Kirtane AJ, Morrow DA, Palabrica TM, Murphy $\mathrm{SA}$, Stone $\mathrm{PH}$, et al. Association between thrombolysis in myocardial infarction myocardial perfusion grade, biomarkers, and clinical outcomes among patients with moderate- to high-risk acute coronary syndromes: Observations from the randomized trial to evaluate the relative PROTECTion against post-PCI microvascular dysfunction and post-PCI ischemia among antiplatelet and antithrombotic agents-Thrombolysis In Myocardial Infarction 30 (PROTECT-TIMI 30). Am Heart J 2006; 152: $756-761$.

21. Nahser PJ Jr, Brown RE, Oskarsson H, Winniford MD, Rossen JD. Maximal coronary flow reserve and metabolic coronary vasodilation in patients with diabetes mellitus. Circulation 1995; 91: $635-640$.

22. De Boer RA, Pinto YM, Van Veldhuisen DJ. The imbalance between oxygen demand and supply as a potential mechanism in the pathophysiology of heart failure: The role of microvascular growth and abnormalities. Microcirculation 2003; 10: 113-126.

23. Morrow DA, Cannon CP, Jesse RL, Newby LK, Ravkilde J, Storrow AB, et al. National Academy of Clinical Biochemistry Laboratory Medicine Practice Guidelines: Clinical characteristics and utilization of biochemical markers in acute coronary syndromes. Circulation 2007; 115: e356-e375.

\section{Supplementary Files}

Please find supplementary file(s);

http://dx.doi.org/10.1253/circj.CJ-18-1196 